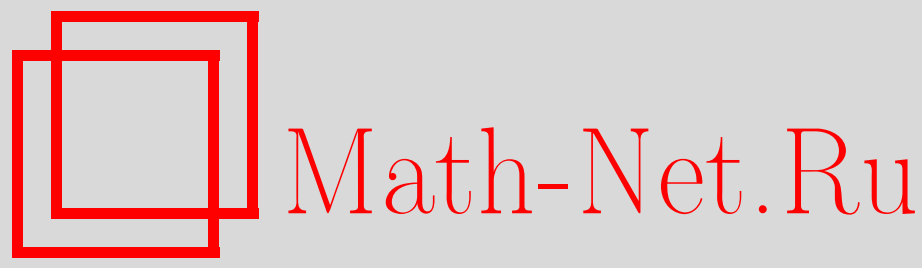

Ю. В. Попов, К. А. Кузаков, А. Булычев, С. И. Виницкий, Теория квазиупругих атомных реакций в переменном электрическом поле, ТМФ, 2016, том 186, номер 1, 113-122

DOI: https://doi.org/10.4213/tmf8980

Использование Общероссийского математического портала Math-Net.Ru подразумевает, что вы прочитали и согласны с пользовательским соглашением http: //www.mathnet.ru/rus/agreement

Параметры загрузки:

IP: 34.229 .108 .108

26 апреля 2023 г., $17: 37: 33$

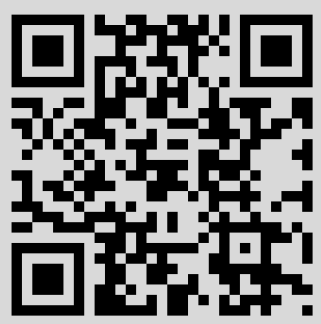




\title{
ТЕОРИЯ КВАЗИУПРУГИХ АТОМНЫХ РЕАКЦИЙ В ПЕРЕМЕННОМ ЭЛЕКТРИЧЕСКОМ ПОЛЕ
}

\begin{abstract}
Для квазиупругих $(e, 2 e)$-атомных реакций в присутствии стоячей электромагнитной волны предлагается вариант теории, которая строится по аналогии со стационарным случаем. Попутно формулируются математические проблемы, которые требуют своего решения для строгого обоснования данной теории.
\end{abstract}

Ключевые слова: квазиупругие $(e, 2 e)$-реакции, переменное электромагнитное поле.

DOI: $10.4213 / \operatorname{tmf} 8980$

\section{1. ВВЕДЕНИЕ}

Квазиупругие реакции в атомной физике, когда быстрый электрон налетает на квантовую мишень (атом, молекулу, тонкую пленку) и выбивает также быстрый электрон, причем угол разлета обоих конечных электронов близок к $90^{\circ}$, позволяют сделать важные заключения о волновой функции выбиваемого электрона в мишени. Метод исследования, когда оба конечных электрона детектируются на совпадение, называется электронной импульсной спектроскопией (ЭИС) квантовой мишени. В кинематике ЭИС $(e, 2 e)$-реакции характеризуются большим переданным импульсом и относительно малым импульсом иона. Впервые простейшее теоретическое обоснование этого метода в рамках первого плосковолнового борновского приближения было дано почти 50 лет назад в работе [1]. Позднее появилось множество, в основном экспериментальных, работ. Сошлемся лишь на книгу [2] и обзор [3],

Работа выполнена при поддержке РФФИ (грант № 14-01-00420_а).

* Научно-исследовательский институт ядерной физики им. Д. В. Скобельцына, Московский государственный университет им. М. В. Ломоносова, Москва, Россия.

E-mail: popov@srd.sinp.msu.ru

$\dagger$ †аборатория теоретической физики им. Н.Н. Боголюбова, Объединенный институт ядерных исследований, Дубна, Московская обл., Россия

‡Физический факультет, Московский государственный университет им. М. В. Ломоносова, Москва, Россия 
в которых подводятся некоторые итоги успешного применения этого метода при исследовании квантовой структуры атомов, молекул и тонких пленок. В настоящее время ЭИС стала рабочим инструментом в квантовой химии.

Несмотря на успехи метода ЭИС, дающего прекрасное совпадение простейшей теории с экспериментом, следует отметить, что даже реакция ионизации атома водорода $\mathrm{H}+e \rightarrow \mathrm{H}^{+}+2 e$ требует для своего описания привлечения теории рассеяния трех заряженных частиц, многие детали которой еще недостаточно хорошо разработаны несмотря на достигнутые в последние годы значительные успехи (см., например, обзор [4]). Очевидно, что для быстрых частиц, участвующих в реакции, борновские приближения являются наиболее адекватным математическим аппаратом теории при выборе подходящего возмущающего потенциала. В то же время использование базиса плоских волн в теориях рассеяния заряженных частиц приводит к известным расходимостям высших борновских приближений, что требует разработки и обоснования процедуры перенормировок соответствующих матричных элементов. В свою очередь, такая процедура требует формулировки общей теории многочастичного кулоновского рассеяния в форме уравнений Липпмана-Швингера. В отношении стационарной теории многочастичного рассеяния обзор подходов и результатов дан в работе [4].

Еще больше трудностей возникает, когда атомная реакция протекает в присутствии электромагнитного поля. Такие процессы описываются временны́м уравнением Шредингера (УШ), что вносит дополнительные теоретические проблемы. Эти проблемы усугубляются, если поле имеет вид монохроматической стоячей волны, действующей в интервале времени $-\infty<t<+\infty$, т. е. затрагивает асимптотическую область. В то же время, если поле имеет не слишком большую интенсивность и частоту (в атомных масштабах), физически очевидно, что соответствующая теория возмущений должна давать адекватное описание ионизационных $(e, 2 e)$-процессов при больших энергиях электронов. От таких атомных реакций в поле можно ожидать получения какой-то новой ценной информации о квантовой структуре мишени. При этом следует учитывать, что электромагнитное излучение модифицирует как электронные состояния облучаемой мишени, так и динамику различных процессов взаимодействия заряженных частиц (электронов, ионов) с мишенью.

Пожалуй, к первым известным теоретическим работам, где исследовалась вероятность ионизации атома под действием переменного электрического поля в рамках борновских приближений, следует отнести работы [5]-[8]. Далее последовало множество работ, книг и обзоров - наступила эпоха экспериментального исследования взаимодействия лазерного и синхротронного электромагнитного излучения с атомами, молекулами и поверхностями.

Проблема теории атомных реакций в присутствии электромагнитного поля состоит в корректном описании динамики системы сталкивающихся частиц на асимптотических расстояниях под воздействием излучения с учетом специфики каждого из взаимодействий: электрона с мишенью, лазерного поля с электроном и лазерного поля с мишенью. Если одно из взаимодействий доминирует, то можно применять подход теории возмущений (см., например, одну из первых работ [9] по обоснованию борновских приближений в задаче о потенциальном рассеянии заряженной частицы в присутствии переменного электромагнитного поля). Однако, если мы изуча- 
ем ионизацию мишени электроном и хотим использовать поле как фон, возможно, усиливающий какие-то стороны самой атомной реакции, то характеристики поля (интенсивность, частота) должны быть таковы, чтобы избежать ионизации мишени самим полем.

Мы не будем глубоко вдаваться в историю развития теории атомных реакций в присутствии поля. В основном усилия авторов большинства работ были направлены на запись таких формул, с помощью которых можно было бы получить численные результаты. Большое число ссылок на такого рода теоретические исследования, в том числе и по интересующим нас $(e, 2 e)$-реакциям, было собрано и всесторонне рассмотрено в обзоре [10].

Альтернативный подход к непертурбативному рассмотрению взаимодействия лазерного поля с атомом дает теория Флоке (см., например, обзор [11] и недавнюю монографию [12]). Ее можно применять к гамильтонианам с периодической зависимостью от времени, и в результате задача сводится к решению системы уравнений, не зависящих от времени (так называемые интегральные уравнения Флоке-Липпмана-Швингера).

И все же в большинстве работ, в которых рассматриваются неупругие процессы при высоких энергиях столкновения, используются борновские приближения временно́й теории возмущений. В недавней работе [13] борновские матричные элементы были записаны для случая квазиупругой $(e, 2 e)$-реакции в поле линейно и эллиптически поляризованной стоячей монохроматической волны слабой интенсивности и относительно низкой частоты. Был получен ряд численных результатов [14]-[16] в сравнении с результатами в отсутствие поля, которые указывают на перспективность подобных исследований для получения нового знания о структуре мишени. Вместе с тем без последовательной математической основы теории атомных реакций в поле соответствие таких результатов физической реальности остается под вопросом, особенно если в расчетах фиксируются тонкие эффекты. В настоящей работе предлагается схема построения такой теории по аналогии со стационарной теорией квазиупругих атомных реакций. Мы не претендуем здесь на решение всех возникающих в рамках обсуждаемой задачи проблем, но указываем узкие места, на которые следует обратить внимание в дальнейшем.

В работе преимущественно используются атомные единицы $\hbar=m_{\mathrm{e}}=e=1$.

\section{2. СТАЦИОНАРНАЯ ТЕОРИЯ КВАЗИУПРУГИХ $(e, 2 e)$-ПРОЦЕССОВ}

Кратко напомним базовую теорию $(e, 2 e)$-процессов в кинематике ЭИС, предполагая в качестве мишени простейшую атомную систему - атом водорода. В стационарной формулировке УШ, описывающее взаимодействие электрона с атомом водорода, выглядит следующим образом:

$$
\left[E+\frac{1}{2} \Delta_{1}+\frac{1}{2} \Delta_{2}+\frac{1}{r_{1}}+\frac{1}{r_{2}}-\frac{1}{r_{12}}\right] \Psi\left(\vec{r}_{1}, \vec{r}_{2} ; E\right)=0 .
$$

Здесь $r_{1}, r_{2}$ - координаты электронов (до симметризации мы выбираем электрон 1 налетающим, а электрон 2 связанным в атоме), $E$ - полная энергия реакции. Ядро 
атома (протон) в силу своей огромной массы предполагается неподвижным в процессе соударений и играет роль центра лабораторной системы координат.

Обозначим через $\vec{p}_{0}, \vec{p}_{\mathrm{s}}$ и $\vec{p}_{\mathrm{e}}$ импульсы соответственно налетающего, рассеяного и выбитого электронов. Тогда баланс энергий выглядит следующим образом:

$$
E=\frac{p_{0}^{2}}{2}+\varepsilon_{0}=\frac{p_{\mathrm{s}}^{2}}{2}+\frac{p_{\mathrm{e}}^{2}}{2}
$$

здесь $\varepsilon_{0}$ - энергия основного состояния атома водорода $\left(\varepsilon_{0}=-0.5\right.$ в атомных единицах). Кинетической энергией ядра пренебрегаем.

Начальное состояние системы (электрон-атом) при больших радиусах $r_{1}$ имеет вид

$$
\Psi_{i}\left(\vec{r}_{1}, \vec{r}_{2} ; E\right)=e^{i \vec{p}_{0} \cdot \vec{r}_{1}} \varphi_{0}\left(r_{2}\right),
$$

где $\varphi_{0}(r)=\sqrt{1 / \pi} e^{-r}-$ волновая функция атома водорода.

При записи интегрального уравнения Липпмана-Швингера, вытекающего из (1), начальное состояние (3) достигается, если положить возмущающий потенциал равным $v_{\text {pert }}=1 / r_{12}-1 / r_{1}$. Тогда уравнение Липпмана-Швингера принимает следующий операторный вид:

$$
|\Psi(E)\rangle=\left|\Psi_{i}(E)\right\rangle+\left(E-H_{i}+i 0\right)^{-1} v_{\text {pert }}|\Psi(E)\rangle, \quad H_{i}=h_{01}+h_{02}+v_{2} .
$$

Далее полезно применить к функции Грина спектральное разложение по базисам кулоновских/плоских волн:

$$
\left(E-H_{i}+i 0\right)^{-1}=\sum_{\beta} \int \frac{d^{3} p}{(2 \pi)^{3}} \frac{\left|\varphi_{\beta}^{-}, \vec{p}\right\rangle\left\langle\vec{p}, \varphi_{\beta}^{-}\right|}{E-\varepsilon_{\beta}-p^{2} / 2+i 0} .
$$

Суммирование по $\beta$ подразумевает суммирование по связанным состояниям кулоновского спектра и интегрирование по континууму. Подставляя разложение (5) в формулу (4), получаем стандартную (точную) амплитуду перехода в конечное состояние $\left\langle\vec{p}, \varphi_{\beta}^{-}\right|$:

$$
T_{\beta}(\vec{p})=\left\langle\vec{p}, \varphi_{\beta}^{-}\left|v_{\text {pert }}\right| \Psi(E)\right\rangle .
$$

Амплитуда в первом борновском приближении по возмущающему потенциалу получается из соотношения (6), если вместо $|\Psi(E)\rangle$ подставить $\left|\Psi_{i}(E)\right\rangle$ :

$$
T\left(\vec{p}_{\mathrm{s}}, \vec{p}_{\mathrm{e}}\right)=\left\langle\vec{p}_{\mathrm{s}}, \varphi^{-}\left(\vec{p}_{\mathrm{e}}\right)\left|v_{1}+v_{12}\right| \vec{p}_{0}, \varphi_{0}\right\rangle
$$

Заметим, что $\left\langle\varphi^{-}\left(\vec{p}_{\mathrm{e}}\right) \mid \varphi_{0}\right\rangle=0$ в силу ортогональности кулоновских функций. Это позволяет несколько упростить выражение для амплитуды процесса ЭИС:

$$
T\left(\vec{p}_{\mathrm{s}}, \vec{p}_{\mathrm{e}}\right)=\left\langle\vec{p}_{\mathrm{s}}, \varphi^{-}\left(\vec{p}_{\mathrm{e}}\right)\left|v_{12}\right| \vec{p}_{0}, \varphi_{0}\right\rangle
$$

Последующее упрощающее допущение обычно следует из замены в выражении (7) кулоновской волны на плоскую. Предполагается, что это возможно сделать при большой энергии испущенного электрона. Хотя это и не совсем так со строго математической точки зрения [4], такое упрощение дает блестящее согласие с экспериментом [17]. Окончательно получаем выражение для плосковолнового матричного 
элемента в первом борновском приближении, которое используется на практике при расчетах квазиупругих реакций:

$$
T\left(\vec{p}_{\mathrm{s}}, \vec{p}_{\mathrm{e}}\right)=\left\langle\vec{p}_{\mathrm{s}}, \vec{p}_{\mathrm{e}}\left|v_{12}\right| \vec{p}_{0}, \varphi_{0}\right\rangle
$$

В то же время формула (6) является основой для различных приближений функции конечного состояния, однако мы на этом останавливаться не будем.

\section{3. КВАЗИУПРУГИЕ РЕАКЦИИ В ПРИСУТСТВИИ ПЕРЕМЕННОГО ЭЛЕКТРОМАГНИТНОГО ПОЛЯ}

Напомним некоторые общие положения. Классические уравнения Максвелла для электромагнитного поля можно записать в терминах скалярного $U(\vec{r}, t)$ и векторного $\vec{A}(\vec{r}, t)$ потенциалов. Эти потенциалы вполне однозначно определяют наблюдаемые характеристики электромагнитного поля: напряженности электрического $\overrightarrow{\mathcal{E}}$ и магнитного $\overrightarrow{\mathcal{H}}$ полей. При этом сами потенциалы определены неоднозначно. Например, два набора потенциалов $\left(\overrightarrow{A^{\prime}}, U^{\prime}\right)$ и $(\vec{A}, U)$, где

$$
\overrightarrow{A^{\prime}}=\vec{A}+\vec{\nabla} f, \quad U^{\prime}=U-\frac{1}{c} \frac{\partial f}{\partial t},
$$

дают одни и те же напряженности электрического и магнитного полей для произвольной функции $f(\vec{r}, t)$. При этом фазовое преобразование волновой функции $\left|\Psi^{\prime}(t)\right\rangle=e^{-(1 / c) f}|\Psi(t)\rangle$ оставляет временно́е УШ инвариантным относительно калибровочного преобразования, т. е. замены в нем нештрихованных операторов на штрихованные.

Прежде всего следует заметить, что по определению напряженность поля связана с его потенциалами следующим образом:

$$
\overrightarrow{\mathcal{E}}(\vec{r}, t)=-\frac{1}{c} \frac{\partial}{\partial t} \vec{A}(\vec{r}, t)-\vec{\nabla} U(\vec{r}, t) .
$$

В так называемой кулоновской калибровке предполагается, что $\operatorname{div} \vec{A}=0$, а также в простейшем исследовании делается физическое предположение о слабой зависимости скалярного потенциала поля от координаты в пределах атомных размеров, $U(\vec{r}, t) \simeq U(0, t)$, которое позволяет пренебречь его градиентом в (9). Это приводит к известному дипольному приближению

$$
\vec{A}(\vec{r}, t) \simeq \vec{A}(0, t)=\vec{A}(t) .
$$

Полагая $\vec{A}(t)=\vec{e} A(t)$, получаем линейно поляризованный вектор-потенциал, где $\vec{e}$ есть единичный вектор поляризации. В таком подходе присутствие электромагнитного поля приводит к замене стационарного УШ (1) его временны́м аналогом

$$
\begin{gathered}
{\left[i \frac{\partial}{\partial t}-\frac{1}{2}\left(-i \vec{\nabla}_{1}+\frac{1}{c} \vec{e} A(t)\right)^{2}-\frac{1}{2}\left(-i \vec{\nabla}_{2}+\frac{1}{c} \vec{e} A(t)\right)^{2}+\right.} \\
\left.+\frac{1}{r_{1}}+\frac{1}{r_{2}}-\frac{1}{r_{12}}\right] \Psi\left(\vec{r}_{1}, \vec{r}_{2} ; t\right)=0 .
\end{gathered}
$$

Данное уравнение удобно переписать в так называемой калибровке длины:

$$
\Psi\left(\vec{r}_{1}, \vec{r}_{2} ; t\right)=e^{-i(1 / c) A(t)\left[\left(\vec{e} \cdot \vec{r}_{1}\right)+\left(\vec{e} \cdot \vec{r}_{2}\right)\right]} \Psi_{\mathrm{L}}\left(\vec{r}_{1}, \vec{r}_{2} ; t\right) .
$$


Тогда из (10) следует уравнение

$$
\begin{aligned}
{\left[i \frac{\partial}{\partial t}\right.} & +\left(\frac{1}{2} \Delta_{1}-\mathcal{E}(t)\left(\vec{e} \cdot \vec{r}_{1}\right)\right)+\left(\frac{1}{2} \Delta_{2}+\frac{1}{r_{2}}-\mathcal{E}(t)\left(\vec{e} \cdot \vec{r}_{2}\right)\right)+ \\
& \left.+\frac{1}{r_{1}}-\frac{1}{r_{12}}\right] \Psi_{\mathrm{L}}\left(\vec{r}_{1}, \vec{r}_{2} ; t\right)=0 .
\end{aligned}
$$

В настоящем исследовании мы задаем электрическое поле в виде стоячей монохроматической волны $\mathcal{E}(t)=F \cos (\omega t+\delta)$. Интенсивность поля в атомных единицах связана с освещенностью $I$ в единицах Вт/ см$^{2}$ следующим образом: $F=\sqrt{I / I_{0}}$, где $I_{0}=3.6 \cdot 10^{16} \mathrm{BT} / \mathrm{cm}^{2}$.

При $t \rightarrow-\infty$ мы полагаем, что налетающий электрон удален достаточно далеко от атома водорода. Это обстоятельство позволяет записать асимптотическое временно́е УШ

$$
\left[i \frac{\partial}{\partial t}+\left(\frac{1}{2} \Delta_{1}-\mathcal{E}(t)\left(\vec{e} \cdot \vec{r}_{1}\right)\right)+\left(\frac{1}{2} \Delta_{2}+\frac{1}{r_{2}}-\mathcal{E}(t)\left(\vec{e} \cdot \vec{r}_{2}\right)\right)\right] \Psi_{L}\left(\vec{r}_{1}, \vec{r}_{2} ; t\right)=0
$$

при возмущающем потенциале $v_{\text {pert }}=1 / r_{1}-1 / r_{12}$.

Уравнение свободного электрона в поле

$$
\left(i \frac{\partial}{\partial t}+\frac{1}{2} \Delta-\mathcal{E}(t)(\vec{e} \cdot \vec{r})\right) \chi_{\vec{p}}(\vec{r}, t)=0
$$

имеет аналитическое решение и определяет так называемую нерелятивистскую волковскую функцию [18]

$$
\chi_{\vec{p}}(\vec{r}, t)=\exp \left\{i\left[-E t+\left(\vec{p}+\frac{1}{c} A(t) \vec{e}\right) \cdot \vec{r}-\alpha_{p} \cos (\omega t+\delta)-\zeta(t)\right]\right\},
$$

где

$$
E=\frac{p^{2}}{2}, \quad \alpha_{p}=(\vec{e} \cdot \vec{p}) \frac{F}{\omega^{2}}, \quad \zeta(t)=\frac{1}{2 c^{2}} \int^{t} d t^{\prime} A^{2}\left(t^{\prime}\right)
$$

Заметим, что в полевых обозначениях $(1 / c) A(t)=-(F / \omega) \sin (\omega t+\delta)$ и, как указано в книге [19], предполагается сколь угодно малое затухание электромагнитной волны при $t \rightarrow \mp \infty$. Таким образом, волковская функция характеризуется параметром (импульсом) $\vec{p}$, переходя при указанном затухании поля в плоскую волну. Отметим свойство полноты системы волковских функций,

$$
\int \frac{d^{3} p}{(2 \pi)^{3}} \chi_{\vec{p}}^{*}\left(\vec{r}^{\prime}, t\right) \chi_{\vec{p}}(\vec{r}, t)=\delta\left(\vec{r}-\vec{r}^{\prime}\right)
$$

и их ортогональность, которая, очевидно, следует из (14). Базис волковских функций эквивалентен базису плоских волн, что делает волковские функции чрезвычайно удобными для использования в теории возмущений. 
Временно́е УШ электрона, находящегося одновременно в поле волны и кулоновском потенциале,

$$
\left(i \frac{\partial}{\partial t}+\frac{1}{2} \Delta+\frac{1}{r}-\mathcal{E}(t)(\vec{e} \cdot \vec{r})\right) \varphi_{\beta}(\vec{r}, t)=0
$$

решать значительно сложнее. Собственно, этим и занимаются на протяжении последних десятилетий исследователи, изучающие спектр состояний электрона (см., например, книгу [12]). В зависимости от частоты и интенсивности поля в такой системе могут происходить переходы между связанными состояниями вплоть до ионизации. Да и сами состояния уже отличаются от состояний кулоновского спектра: даже при слабом поле монохроматической волны они смещаются, могут перемешиваться. Классификацию таких состояний в слабом поле и поиск низшего (основного) состояния с индексом 0 следует производить в рамках теории Флоке.

Допустим, мы провели такую классификацию по некоторому параметру $\beta$. Нетрудно показать (потребовать) ортогональность этих состояний,

$$
\int d^{3} r \varphi_{\beta}^{*}(\vec{r}, t) \varphi_{\beta^{\prime}}(\vec{r}, t)=\delta_{\beta, \beta^{\prime}},
$$

сложнее показать свойство полноты системы функций $\varphi_{\beta}(\vec{r}, t)$,

$$
\sum_{\beta} \varphi_{\beta}^{*}\left(\vec{r}^{\prime}, t\right) \varphi_{\beta}(\vec{r}, t)=\delta\left(\vec{r}-\vec{r}^{\prime}\right)
$$

которое необходимо для разложения соответствующей функции Грина в ряд/интеграл по параметру $\beta$ и извлечения из этого разложения амплитуд рассеяния. Несмотря на ряд интересных соображений на данную тему, приведенных в некоторых немногочисленных работах (см., например, работу [20], формулы (16)-(23)), этот вопрос, на наш взгляд, остается малоизученным.

Однако допустим, что мы нашли спектр уравнения (16) и доказали свойство полноты спектральных функций хотя бы при слабых полях. Тогда из (12) следует интегральное уравнение

$$
\begin{aligned}
\left|\Psi_{\mathrm{L}}(t)\right\rangle= & \left|\varphi_{0}^{-}(t), \chi_{\vec{p}_{0}}(t)\right\rangle+ \\
& +i \sum_{\beta} \int \frac{d^{3} p}{(2 \pi)^{3}}\left|\varphi_{\beta}^{-}(t), \chi_{\vec{p}}(t)\right\rangle \int_{-\infty}^{t} d t^{\prime}\left\langle\chi_{\vec{p}}\left(t^{\prime}\right), \varphi_{\beta}^{-}\left(t^{\prime}\right)\left|v_{\text {pert }}\right| \Psi_{\mathrm{L}}\left(t^{\prime}\right)\right\rangle .
\end{aligned}
$$

Таким образом можно определить амплитуду перехода в произвольный конечный канал по аналогии с выражением (6):

$$
T_{\beta}(\vec{p})=\int_{-\infty}^{\infty} d t^{\prime}\left\langle\chi_{\vec{p}}\left(t^{\prime}\right), \varphi_{\beta}\left(t^{\prime}\right)\left|v_{\text {pert }}\right| \Psi_{\mathrm{L}}\left(t^{\prime}\right)\right\rangle
$$


В первом борновском приближении мы имеем

$$
\begin{aligned}
T\left(\vec{p}_{\mathrm{s}}, \vec{p}_{\mathrm{e}}\right) & =\int_{-\infty}^{\infty} d t^{\prime}\left\langle\chi_{\vec{p}_{\mathrm{s}}}\left(t^{\prime}\right), \varphi_{\vec{p}_{\mathrm{e}}}\left(t^{\prime}\right)\left|v_{\text {pert }}\right| \varphi_{0}\left(t^{\prime}\right), \chi_{\vec{p}_{0}}\left(t^{\prime}\right)\right\rangle= \\
& =\int_{-\infty}^{\infty} d t^{\prime}\left\langle\chi_{\vec{p}_{\mathrm{s}}}\left(t^{\prime}\right), \varphi_{\vec{p}_{\mathrm{e}}}\left(t^{\prime}\right)\left|v_{12}\right| \varphi_{0}\left(t^{\prime}\right), \chi_{\vec{p}_{0}}\left(t^{\prime}\right)\right\rangle .
\end{aligned}
$$

Последнее равенство в (20) следует из ортогональности функций $\left|\varphi_{\beta}(t)\right\rangle$. Естественно, возникает вопрос, какие подводные камни имеются в борновском разложении матричного элемента (19)? Отметим, что интегрирование по времени, вообще говоря, не гарантирует отсутствие перекрывания особенностей в функции Грина и потенциалах в (19), которое, напомним, приводит к сингулярностям интегралов при разложении (6) в борновский ряд. Здесь требуется проведение соответствующего строгого математического исследования.

Наконец, при больших квантовых числах $\vec{p}$, имеющих смысл импульса электрона, делается приближение $\left|\varphi_{\vec{p}_{\mathrm{e}}}(t)\right\rangle \approx\left|\chi_{\vec{p}_{\mathrm{e}}}(t)\right\rangle$. Его можно попытаться обосновать путем использования приближения сильного поля, применимость которого, однако, не до конца исследована при низкой частоте.

Расчеты в приближении (20) с тремя волковскими функциями, описывающими электроны в континууме, представлены в работах [13]-[16]. Если поле отсутствует $(F=0)$, результат пространственного интегрирования в формуле $(20)$ совпадает с выражением (7), а интегрирование по времени дает дельта-функцию, отвечающую закону сохранения энергии. С техникой расчетов, результаты которых в настоящее время используются при подготовке первых экспериментов с использованием ЭИС в присутствии лазерного поля, можно ознакомиться в указанных работах. Сама установка создается в Университете Тохоку (Сендай, Япония) [21], [22].

\section{4. ОБСУЖДЕНИЕ}

Рассмотрим слабые места теории атомных реакций в поле монохроматической электромагнитной волны и кратко обсудим возможные пути их преодоления.

\section{1. Классификация решений уравнения (16) и полнота (17) системы}

таких решений. Известно, что в поле стоячей монохроматической волны можно провести классификацию состояний по так называемой квазиэнергии $\mathbb{E}_{j}$ в рамках теории Флоке [12], [23]-[25].

Что касается дискретного спектра, квазиэнергия получается из собственной энергии $\varepsilon_{j}$ связанного состояния атома водорода. Имеет место формула $\mathbb{E}_{j}=\varepsilon_{j}+\delta_{j}+n_{j} \omega$, где $\delta_{j}$ - динамический штарковский сдвиг и $n_{j}-$ число фотонов.

Состояния непрерывного спектра, как и в случае волковских функций, характеризуются импульсом и связанной с ним энергией. Действительно, волновая функция непрерывного спектра уравнения (16) при соответствующей асимптотике может переходить в волковскую функцию (14), если убрать кулоновский потенциал. К сожалению, аналитическое решение уравнения (16) в случае непрерывного спектра 
неизвестно, но есть ряд приближенных выражений: очевидное включение логарифмической кулоновской фазы (сходящейся и расходящейся) в экспоненту в выражении (14), умножение волковской функции на искажающие факторы, присущие кулоновской волне [26]-[29] (так называемые кулоновско-волковские волны) и различные квазиклассические поправки в рамках эйконально-волковских состояний (см., например, работы [30]-[32]).

В принципе можно показать, что такая классификация дает полный набор собственных функций, хотя это требует строгих математических доказательств.

\section{2. Расходимости членов борновского ряда, генерируемых выражени-} ем (19). Напомним, что расходимость высших членов борновского ряда в стационарном случае связана с перекрыванием интегрируемой сингулярности кулоновского потенциала сингулярностью свободной функции Грина в случае, когда частицы подчиняются закону сохранения энергии во входном/выходном каналах. Сложная зависимость функций в уравнении (18) и выражении (19) от времени, по которому проводится интегрирование, возможно, позволяет избежать расходимостей высших борновских членов в этом случае, но до настоящего времени данный вопрос не исследовался, поскольку на практике, как правило, расчеты не идут дальше первого борновского члена (20). Отметим, что в недавних работах [33], [34], претендующих на проведение расчетов в рамках второго борновского приближения, проблема возможных расходимостей не обсуждается.

\section{3. Справедливость приближения $\left|\varphi_{\vec{p}_{\mathrm{e}}}(t)\right\rangle \approx\left|\chi_{\vec{p}_{\mathrm{e}}}(t)\right\rangle$ при больших им-} пульсах $p_{\mathrm{e}}$. Это чисто техническое физическое приближение обычно делают для облегчения расчетов, и оно равносильно приближению кулоновской волны плоской. Все схемы в рамках приближения сильного поля носят скорее интуитивный, чем математический, характер, и возможны не при всех частотах и интенсивностях.

И еще одно заключительное замечание об общем случае квантовой реакции в присутствии электромагнитного поля. В отсутствие поля в теории рассеяния заряженных частиц важную роль играет так называемое асимптотическое условие Долларда [35], определяющее совпадение асимптот квантового и классического движения заряженных частиц. Опираясь на асимптотические соображения, С. П. Меркурьев сформулировал основные положения задачи рассеяния трех заряженных частиц (см., например, монографию [36]). Стоячая монохроматическая волна присутствует в асимптотике, и тем самым требуется корректная математическая переформулировка асимптотического условия, что является достойной математической задачей. С волнами конечной длительности ситуация несколько проще, так как электромагнитное поле отсутствует при $t \rightarrow \pm \infty$.

\section{5. ЗАКЛЮЧЕНИЕ}

В работе сформулированы математические проблемы, требующие своего решения для строгого обоснования теории квазиупругих атомных реакций в присутствии электромагнитного поля стоячей волны.

Благодарности. Авторы благодарны А. К. Мотовилову за идею работы и ценные замечания. 
Ю. В. ПОПОВ, К.А. КУЗАКОВ, А. А. БУлыЧЕВ, С. И. ВИНИцКИй

\section{Список литературы}

[1] Ю. Ф. Смирнов, В. Г. Неудачин, Писъма в ЖЭТФ, 3:7 (1966), 298-301.

[2] E. Weigold, I. E. McCarthy, Electron Momentum Spectroscopy, Kluwer, New York, 1999.

[3] В. Г. Неудачин, Ю. В. Попов, Ю. Ф. Смирнов, УФН, 169:10 (1999), 1111-1139.

[4] В. Л. Шаблов, П. С. Виницкий, Ю. В. Попов, О. Чулуунбаатар, К. А. Кузаков, ЭЧАЯ, 41:2 (2010), 607-650.

[5] Л. В. Келдыш, ЖЭТФ, 47:5 (1965), 1945-1957.

[6] А. М. Переломов, В. С. Попов, М. В. Терентьев, ЖЭТФ, 50:5 (1966), 1393-1409.

[7] А. М. Переломов, В. С. Попов, ЖЭТФ, 50:1 (1966), 179-198.

[8] А. М. Переломов, В. С. Попов, М. В. Терентьев, ЖЭТФ, 51:1 (1967), 309-326.

[9] N. M. Kroll, K. M. Watson, Phys. Rev. A, 8:2 (1973), 804-809.

[10] F. Ehlotzky, A. Jaro, J. Z. Kaminski, Phys. Rep., 297:2-3 (1998), 63-153.

[11] S.-I. Chu, "Recent developments in semiclassical Floquet theories for intense-field multiphoton processes", Advances in Atomic and Molecular Physics, 21, eds. D. R. Bates, B. Bederson, Academic Press, New York, London, 1985, 197-253.

[12] C. J. Joachain, N. J. Kylstra, R. M. Potvliege, Atoms in Intense Laser Fields, Cambridge Univ. Press, Cambridge, 2011.

[13] K. A. Kouzakov, Yu. V. Popov, M. Takahashi, Phys. Rev. A, 82:2 (2010), 023410, 14 pp.

[14] A. A. Bulychev, K. A. Kouzakov, Yu. V. Popov, Phys. Lett. A, 376:4 (2012), 484-487.

[15] A. A. Bulychev, K. A. Kouzakov, Eur. Phys. J. D, 68:11 (2014), 354, 7 pp.

[16] A. A. Bulychev, K. A. Kouzakov, Phys. Rev. A, 91:2 (2015), 023413, 8 pp.

[17] B. Lohmann, E. Weigold, Phys. Lett. A, 86:3 (1981), 139-141.

[18] D. M. Wolkow, Z. Phys., 94:3-4 (1935), 250-260.

[19] В. М. Берестецкий, Е. М. Лифшиц, Л.П. Питаевский, Релятивистская квантовая теория, т. 1, Наука, М., 1968.

[20] F. H. M. Faisal, J. Phys. B, 40:7 (2007), F145-F155.

[21] M. Yamazaki, Y. Kasai, K. Oishi, H. Nakazawa, M. Takahashi, Rev. Sci. Instrum., 84:6 (2013), 063105, $10 \mathrm{pp}$.

[22] Y. Masakazu, M. Yamazaki, K. Oishi, H. Nakazawa, C.-Y. Zhu, M. Takahashi, Phys. Rev. Lett., 114:10 (2015), 103005, 5 pp.

[23] M. Dörr, C. J. Joachain, R. M. Potvliege, S. Vučić, Phys. Rev. A, 49:6 (1994), 4852-4863.

[24] R. Shakeshaft, X. Tang, Phys. Rev. A, 36:7 (1987), 3193-3202.

[25] X. Tang, R. Shakeshaft, Z. Phys. D, 5:1 (1987), 27-34.

[26] P. Krstić, M. H. Mittleman, Phys. Rev. A, 44:9 (1991), 5938-5946.

[27] J.Z. Kamiński, A. Jaroń, F. Ehlotzky, Phys. Rev. A, 53:3 (1996), 1756-1761.

[28] G. Duchateau, E. Cormier, R. Gayet, Phys. Rev. A, 66:2 (2002), 023412, 7 pp.

[29] F.H. M. Faisal, "Atomic multi-photon interaction with intense short-wavelength fields", Strong Field Laser Physics, Springer Series in Optical Sciences, 134, ed. T. Brabec, Springer, New York, 2009, 391-424.

[30] J. I. Gersten, M. H. Mittleman, Phys. Rev. A, 12:5 (1975), 1840-1845.

[31] V.P. Krainov, J. Opt. Soc. Amer. B, 14:2 (1997), 425-431.

[32] S. Gordienko, J. Meyer-ter-Vehn, SPIE Proc., 5228 (2003), 416-426.

[33] M.-Y. Zheng, S.-M. Li, Phys. Rev. A, 82:2 (2010), 023414, 7 pp.

[34] I. Ajana, A. Makhoute, D. Khalil, A. Dubois, J. Phys. B, 47:17 (2014), 175001.

[35] J. D. Dollard, J. Math. Phys., 5:6 (1964), 729-738.

[36] С. П. Меркурьев, Л. Д. Фаддеев, Квантовая теория рассеяния для систем нескольких частии, Наука, М., 1985. 